\title{
ANÁLISIS DE ILUMINACIÓN EN AMBIENTES DE APRENDIZAJE VIRTUAL EN ESTUDIANTES DE LA UNIVERSIDAD PRIVADA DE TACNA DURANTE CONFINAMIENTO POR COVID-19
}

\author{
ANALYSIS OF LIGHTING IN VIRTUAL LEARNING ENVIRONMENTS IN STUDENTS OF THE PRIVATE \\ UNIVERSITY OF TACNA DURING CONFINEMENT BY COVID-19.
}

\author{
Gabriela Alexandra Vera Mazuelos ${ }^{1}$ \\ https://orcid.org/0000- 0001-7241-784X \\ arq.gabrielavera@outlook.com \\ Gabriela Isabel Heredia Alvarez ${ }^{1}$ \\ https://orcid.org/0000-0002-1649-0206 \\ gabitahere@yahoo.com \\ Pablo Enrique Mazuelos Soldevilla ${ }^{1}$ \\ https://orcid.org/0000-0002-7856-8785 \\ pablo.mazuelos.s@gmail.com \\ Yeshua Huallpa Condori ${ }^{1}$ \\ https://orcid.org/0000-0003-0396-1934 \\ emmanuelhuallpa@gmail.com
}

\begin{abstract}
RESUMEN
La investigación se realizó con el propósito de determinar sí, el ambiente de estudio utilizado por los estudiantes de la Universidad Privada de Tacna durante el confinamiento por COVID-19, cumple con los requerimientos lumínicos necesarios para garantizar un espacio saludable, contemplando las regulaciones e índices de iluminación normados. La metodología estuvo enmarcada dentro del tipo de investigación aplicada, cuya población de estudio fue el cuerpo estudiantil de la Universidad Privada de Tacna con una muestra de 222 estudiantes a quienes se aplicó una encuesta de 20 preguntas. El método de análisis lumínico de ambientes de estudio con relación al cumplimiento de la norma técnica EM.010 Instalaciones eléctricas interiores del Reglamento Nacional de Edificaciones (anexo: requisitos mínimos de iluminación) se realizó en 7 viviendas; empleándose un
\end{abstract}

\footnotetext{
1 Universidad Privada de Tacna.
} 
luxómetro marca Extech modelo 407026. Los resultados encontrados indican que en ningún momento del día se alcanza los índices recomendados de $5001 x$ y que los índices encontrados en los ambientes de estudio están por debajo de lo establecido. Se concluye que en todos los casos es necesario adicionar una luminaria con iluminación dirigida al plano de trabajo de condiciones cercanas a 1800 lúmenes, 6500K y $18 \mathrm{w}$.

Palabras clave: Iluminación, espacio saludable, entornos de aprendizaje, COVID-19.

\section{ABSTRACT}

The research was carried out with the purpose of determining whether the study environment used by the students of the Private University of Tacna during the confinement by COVID-19, complies with the light requirements necessary to guarantee a healthy space, considering the regulations and indexes lighting standards. The methodology was framed within the type of applied research, whose study population was the student body of the Private University of Tacna with a sample of 222 students to whom a survey of 20 questions was applied. The method of light analysis of study environments in relation to compliance with the technical standard EM.010 Indoor electrical installations of the National Building Regulations (annex: minimum lighting requirements) was carried out in 7 homes; using an Extech model 407026 lux meter. The results found indicate that the recommended rates of 500lx are not reached at any time of the day and that the rates found in the study environments are below what is established. It is concluded that in all case it is necessary to add a luminaire with lighting directed to the work plane with conditions close to 1800 lumens, $6500 \mathrm{~K}$ and $18 \mathrm{w}$.

Keywords: Lighting, healthy space, learning environments, virtualclasses, COVID-19.

\section{INTRODUCCIÓN}

Uno de los factores imprescindibles de un espacio es la iluminación, sea natural o artificial, la luz tiene la capacidad de cualificar los espacios, puede hacer que un espacio se sienta más acogedor o agobiante a través de sus diferentes cualidades como intensidad lumínica, cromatismo, oscilaciones, e incluso la orientación del edificio para una adecuada iluminación natural, son factores determinantes en el diseño del espacio. Es indiscutible la influencia de la iluminación dentro de nuestras funciones biológicas y psicológicas. Sabemos que la iluminación es un sincronizador del ritmo circadiano; que también es un condicionante para la percepción del color, la agudeza visual; influye en nuestro estado de ánimo y desempeño de ciertas actividades. La iluminación monótona y deficiente de un espacio que habitemos con regularidad puede causar falta de atención, desanimo, incremento del estrés, fatiga y depresión. Sin embargo, el uso de una iluminación adecuada puede tener resultados positivos, lo que hace que la iluminación sea parte esencial dentro del diseño para crear 
edificios más saludables y se deba tener en cuenta dentro de los espacios dedicados al aprendizaje.

En cuanto a la influencia de la iluminación en los estudiantes, el uso adecuado de dentro del aula puede contribuir a la mejora del rendimiento, razón por la que se han realizado múltiples estudios para analizar el efecto de la iluminación dentro de las aulas. Los resultados de estudios como: (Lee et al., 2012); (Marchand et al., 2014); (Samani et al., 2012); (Burruss, 2001) determinaron la importancia e influencia de la iluminación en espacios de aprendizaje y su relación directa con el desempeño de los estudiantes, demostrado que, a través de variaciones en la iluminación artificial se puede influir en la agudeza visual y en las tareas de concentración (Cachán et al., 2012). Otro factor de análisis es la temperatura de color emitida por las fuentes de luz, que tiene gran importancia en el comportamiento de los alumnos y su aprovechamiento escolar (Hartstein et al., 2018). El uso de luminarias con temperatura de color dentro del rango de $6000 \mathrm{~K}$ a $10000 \mathrm{~K}$, proporciona un ambiente similar a la luz del día, que ayuda a evitar la sensación de fatiga que pueden sufrir algunos alumnos por la permanencia de varias horas en un recinto cerrado, estimulando su concentración, mientras que el uso de luminarias con temperatura de color dentro del rango de $1000 \mathrm{~K}$ a $5000 \mathrm{~K}$ dan lugar a ambientes más sociables y relajados.

Con estas referencias en la investigación podemos verificar que luz artificial tiene una importante influencia dentro de los espacios destinados al aprendizaje. En este contexto, existen normas nacionales e internaciones que especifican requerimientos lumínicos que garantizan las condiciones de iluminación necesarias para el desarrollo de cada actividad, según sus necesidades. Dentro de nuestra normativa peruana el Sub-Título III.4, Instalaciones eléctricas y mecánicas del RNE (EM-010, 2019), especifica la iluminancia en el servicio (Lux) según cada ambiente, especificando 500 Lux para ambientes de clases, laboratorio, talleres y gimnasios, por otro lado, los requerimientos dentro de una vivienda varían por debajo de los 300 Lux.

\section{MATERIALES Y MÉTODOS}

La investigación se enmarca en la siguiente clasificación: De acuerdo con los objetivos es del tipo de investigación aplicada, porque el problema parte de la identificación de las características de los ambientes de estudio a través de la aplicación de un cuestionario a la población de estudio y el análisis lumínico con relación al cumplimiento de la norma técnica EM.010 Instalaciones eléctricas interiores del Reglamento Nacional de Edificaciones (anexo: requisitos mínimos de iluminación) de ambientes seleccionados de la población total estudiada. Por el nivel de investigación es descriptiva, ya que, se pretende describir, caracterizar y analizar la iluminación en el entorno físico de aprendizaje. Así mismo, la investigación descriptiva opera cuando se requiere delinear las características específicas descubiertas por las investigaciones exploratorias. Esta descripción podría realizarse usando métodos cualitativos y en un estado superior de descripción, usando métodos cuantitativos. En cuanto al diseño de la investigación se consideró un diseño no experimental al analizar las condiciones lumínicas presentes en el espacio sin mayor modificación; sin embargo, se realizó una simulación digital para representar un escenario con la integración de una mejora, desde un punto de vista general, se refieren al diseño como un "plan o estrategia que se desarrolla para obtener la información que se requiere en una investigación" (Niño, 2011). El propósito 
de la investigación describió el entorno físico en el cual los estudiantes de la Universidad Privada de Tacna desarrollan sus procesos de aprendizaje de manera virtual.

Habiendo entendido las técnicas como los procedimientos específicos (conjunto de mecanismos, medios o recursos) para recoger la información o los datos requeridos, así como como instrumentos y materiales que necesita preparar el investigador en la aplicación de cada técnica. Las técnicas tomadas en cuenta para la recopilación de información fueron: De fuentes primarias, documentos normativos que regulan las condiciones de los espacios de aprendizaje, así como tesis y artículos científicos relacionados al tema. De fuentes secundarias, se recopiló información de estudios realizados en otros ámbitos a nivel internacional. Esta información sirvió como guía en los procesos de estudio de caracterización, así como en los resultados obtenidos de las encuestas.

El instrumento aplicado fue la encuesta virtual, para recopilar información de primera fuente en estudiantes de las diferentes carreras de la Universidad Privada de Tacna. El cuestionario comprendió tres secciones, la primera presenta el tema y sus objetivos, la segunda contiene preguntas simples (edad, género, carrera profesional, ciclo en el que se encuentra matriculado, rango de horario en el cual realiza las actividades lectivas), la última parte incluye preguntas sobre la descripción del espacio área de estudio como; tipo de iluminación artificial, temperatura de color, iluminación natural y cantidad y ubicación de elementos de iluminación dentro del es pacio: 1, 2, 3 o más.

Respecto al análisis de los espacios seleccionados aleatoriamente para medición: se han tomado en consideración 7espacios de estudio, debido a las condiciones cuarentenarias de estricto cumplimiento en las cuales sellevó a cabo la investigación. En estos espacios se realizó la medición de iluminación en el área de trabajo, según el horario de clases. La medida utilizada de acuerdo al sistema internacional de unidades es: Lux (Ix) definida como iluminancia que es la cantidad de flujo luminoso que incide sobre una superficie por unidad de área. La medida fue tomada con la computadora encendida y con el equipo luxómetro de marca Extech Instruments, modelo: 407026, Sensor: $3.3 \times 2.2 \times 0.7$ " ( $85 \times 55 \times 17.5 \mathrm{~mm})$. Se comparó la condición de iluminación actual de los participantes, la iluminación requerida y la iluminación con la implementación de una lámpara de iluminación dirigida al plano de trabajo. Dentro del escenario propuesto en el modelo 3D en el programa de simulación de iluminación DiaLUX, se ha tenido en consideración la implementación de una lámpara de mesa con un foco LED de 1800 lúmenes de potencia, 6500k y 18w.

Para el procesamiento de datos obtenidos de la encuesta del presente trabajo de investigación se realizó mediante la aplicación del software estadístico SPSS. La presentación de la información se realizó a través de cuadros estadísticos y para su análisis mediante datos porcentuales. El proceso de los datos obtenidos de las mediciones realizadas en los espacios seleccionados y los escenarios producidos en el programa de simulación de iluminación DiaLux, se presentarán a través de cuadros para su análisis. La discusión de los datos se realizó teniendo en cuenta el marco teórico referente al tema

\section{RESULTADOS}

Los elementos de estudio que influyen en el cumplimiento de la norma técnica EM.010 Instalaciones eléctricas interiores del Reglamento Nacional de Edificaciones (anexo: requisitos mínimos de iluminación), son elementos referidos a las propiedades lumínicas de las luminarias presentes en el espacio de estudio. Así mismo, para la demostración de la hipótesis 
se ha considerado las medidas de la iluminación (Lux) en los planos de trabajo destinados a las actividades lectivas dentro de los espacios analizados en campo, determinándose que en ningún caso se cumplen los parámetros indicados en la norma correspondientes a 500 Luxes. En tal sentido, se rechaza la hipótesis y aceptamos la hipótesis alternativa que indica que los valores están por debajo de la norma. Esto es los espacios de estudio de los alumnos de la Universidad Privada de Tacna no cumplen con los parámetros lumínicos necesarios para el desarrollo de sus actividades lectivas. El déficit lumínico presente es de $71.4 \%$ considerando la medición promedio en los diferentes espacios de análisis 147 Lx. y siendo 500 Lx la medida mínima para la realización de actividades lectivas.

Los estudiantes, ante un escenario de actividades presenciales, acudían a las instalaciones de la universidad teniendo áreas y servicios adecuados para el desarrollo de sus actividades lectivas, actividades de estudio y complementaria. Espacios regulados por el RNE, velando por que los estudiantes tengan las condiciones adecuadas para realizar sus actividades lectivas, sin embargo, son condiciones que no están reguladas dentro del hogar. Es especialmente interesante comparar este cambio en la modalidad de enseñanza y aprendizaje de los estudiantes. Como se muestra en la Fig. 1 el $73.4 \%$ de los estudiantes realizaron modificaciones para adaptar su ambiente de estudio; un $23.9 \%$ refiere que no hizo modificaciones y solo un $2.7 \%$ no sabe o no responde. En la Fig. 2 se puede observar que, el $84.2 \%$ de los estudiantes, manifiestan que los focos se encuentran ubicados en el techo; solo un $9.5 \%$ refiere que se encuentran tanto en el techo como en su mesa de trabajo. Mostrando que en muy pocos casos se considera una iluminación dirigida al plano de trabajo.

Figura 1

Hizo modificaciones para adaptar el ambiente de estudio.

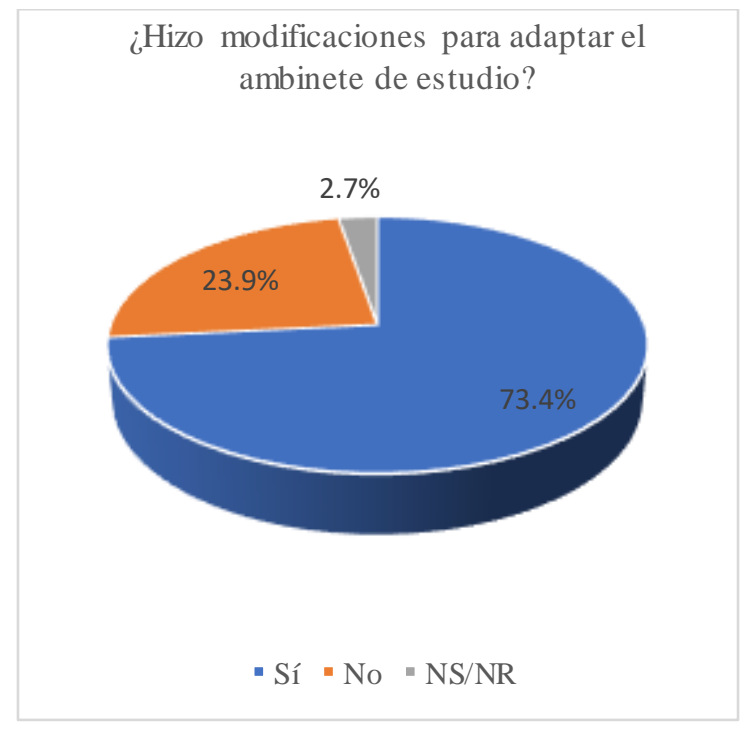

Figura 2

Ubicación de los focos en el ambiente de estudio

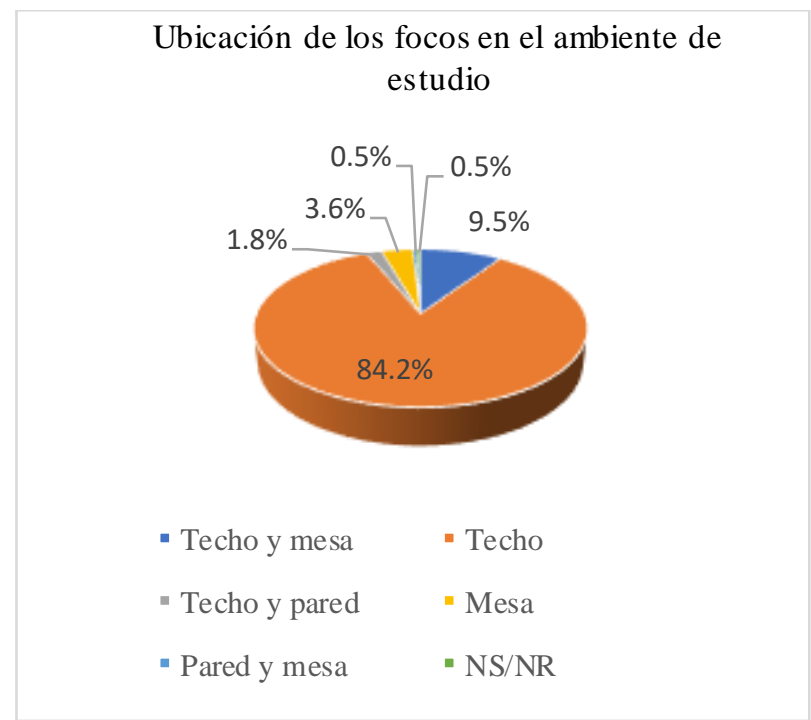

Respecto al análisis los es pacios seleccionados para medición, como se observa en la Figura- 3, la media de iluminación en los horarios de mañana, tarde y noche, de los 7 casos de 
estudio, muestra que en ningún momento del día se alcanza los índices recomendados de $500 l x$ y que los índices mostrados están muy por debajo de lo recomendado.

Figura 3 lluminación promedio por rango horario

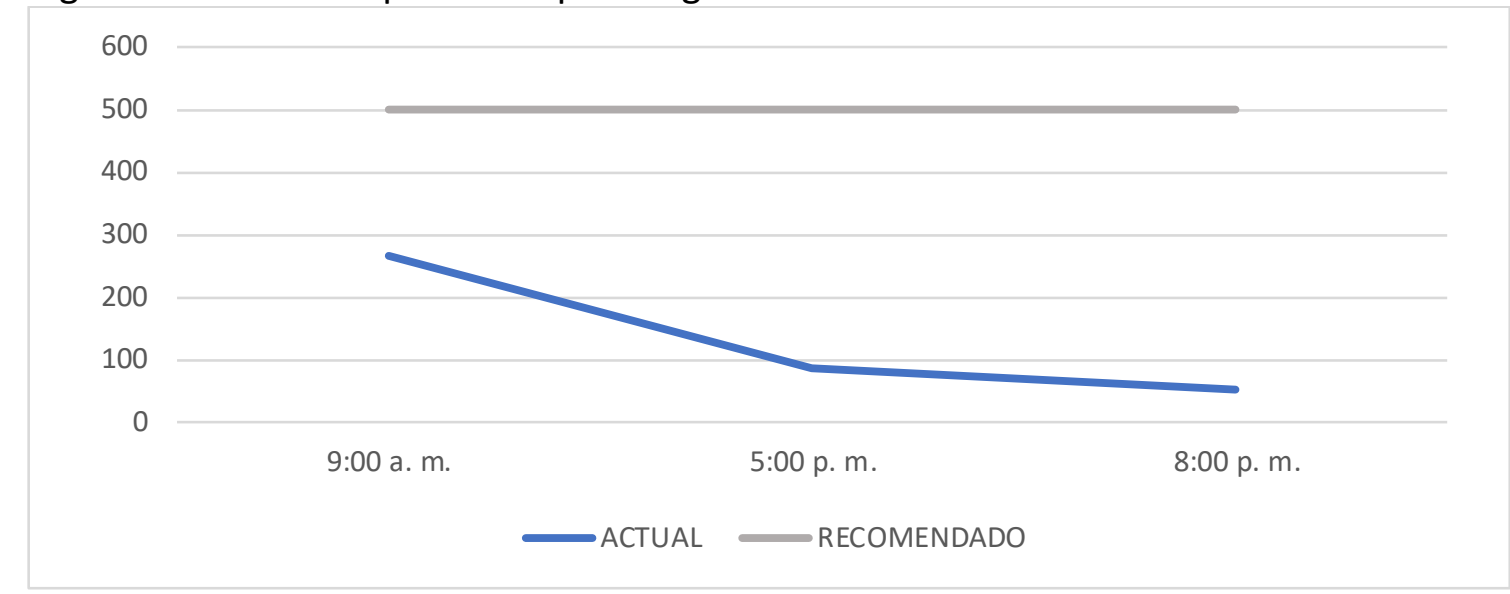

Como se observa en la Figura 4, de los estudiantes que realizan sus actividades en la mañana, tomando como referencia las 9:00 AM como horario de medición, ninguno cumple con las condiciones de $5001 x$.

\section{Figura 4}

Iluminación promedio en la mañana 9:00 am

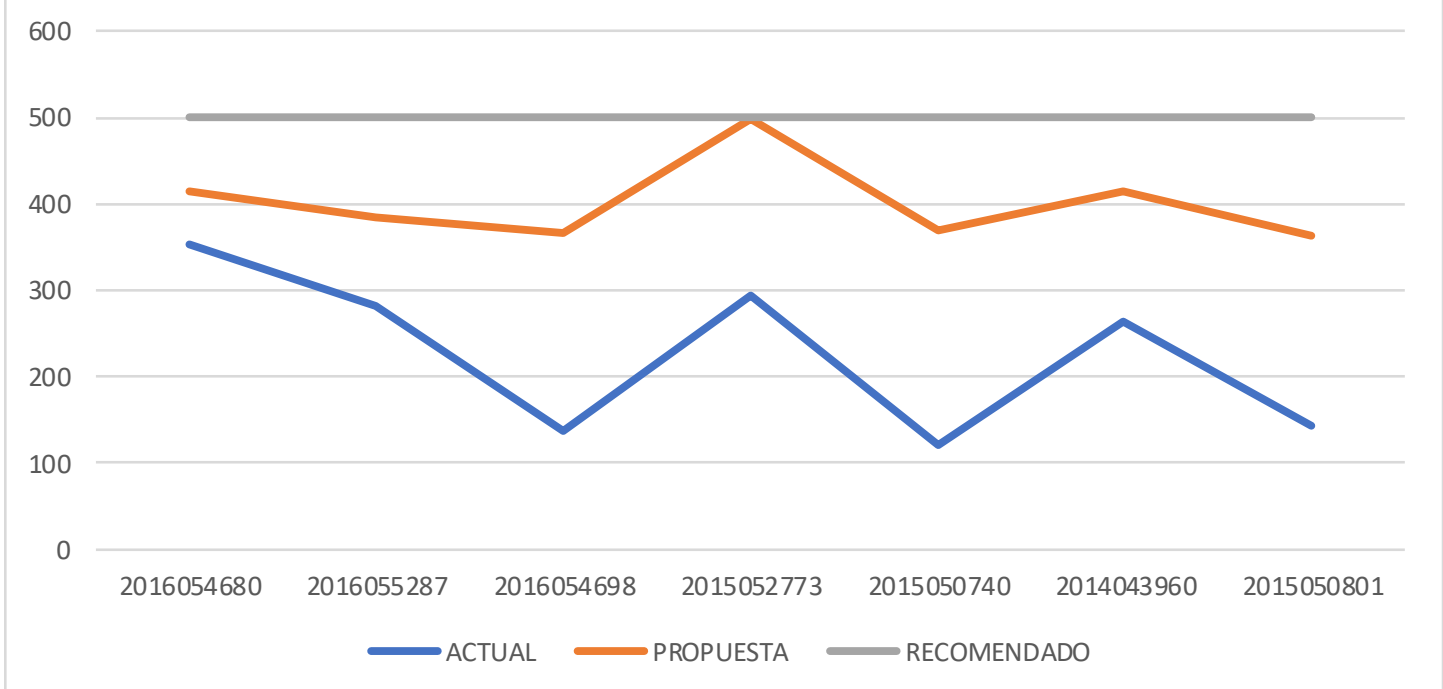

En la Figura 5, de los estudiantes que realizan sus actividades en la tarde, tomando como referencia las 5:00 PM como horario de medición, ninguno cumple con las condiciones de 500lx. Sin embargo, con la implementación de una lampara de mesa, con las especificaciones mencionadas anteriormente, existe una importante mejora y en dos casos sobrepasaría los 500lx recomendados. Se tiene en cuenta que el caso 2014043960 tiene una lampara de mesa, por la cual la mejora es muy poco significativa. 
Figura 5

Iluminación promedio en la tarde 5:00 pm

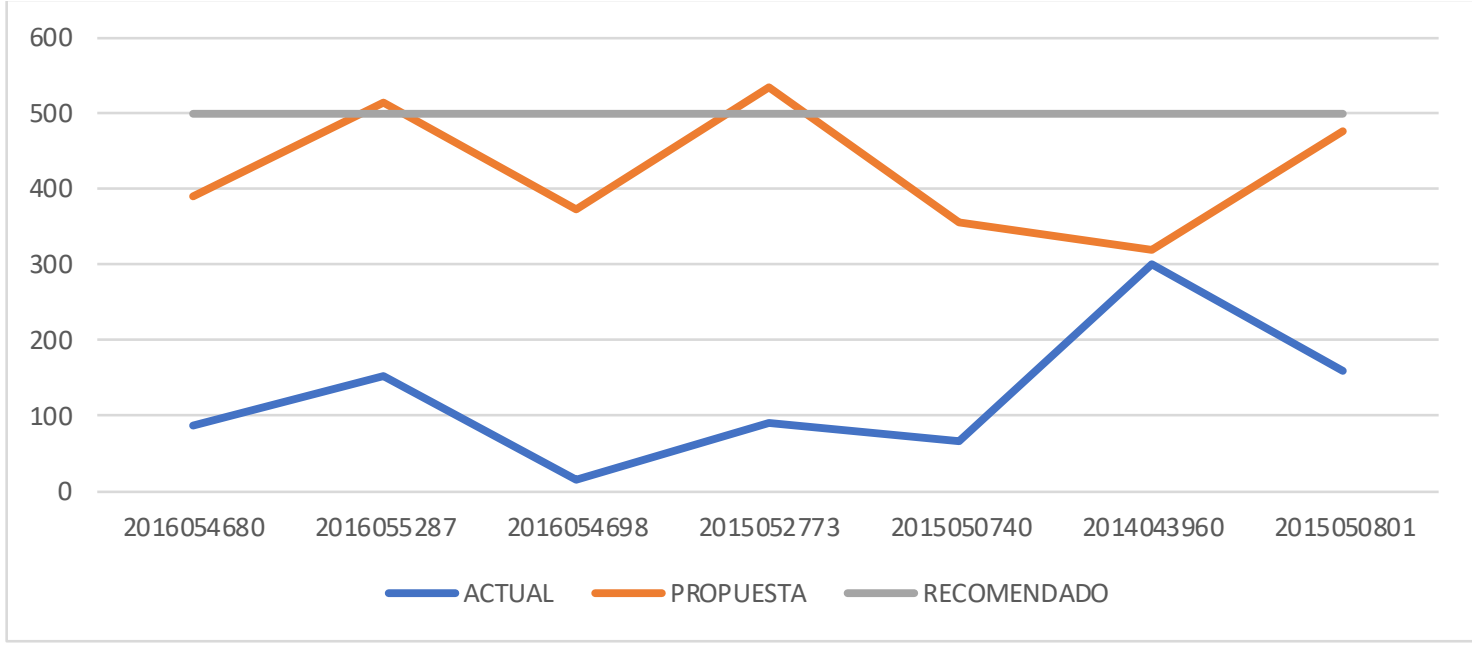

En la figura 6, de los estudiantes que realizan sus actividades en la tarde, tomando como referencia las 8:00 PM como horario de medición, ninguno cumple con las condiciones de 500lx, estando muy por debajo de lo recomendado. Sin embargo, en el escenario propuesto con la implementación de una lampara de mesa, existe una importante mejora aunque en ninguno de los casos llega a los 500lx recomendados. Se tiene en cuenta que el caso 2014043960 es el único que tiene una lampara de mesa.

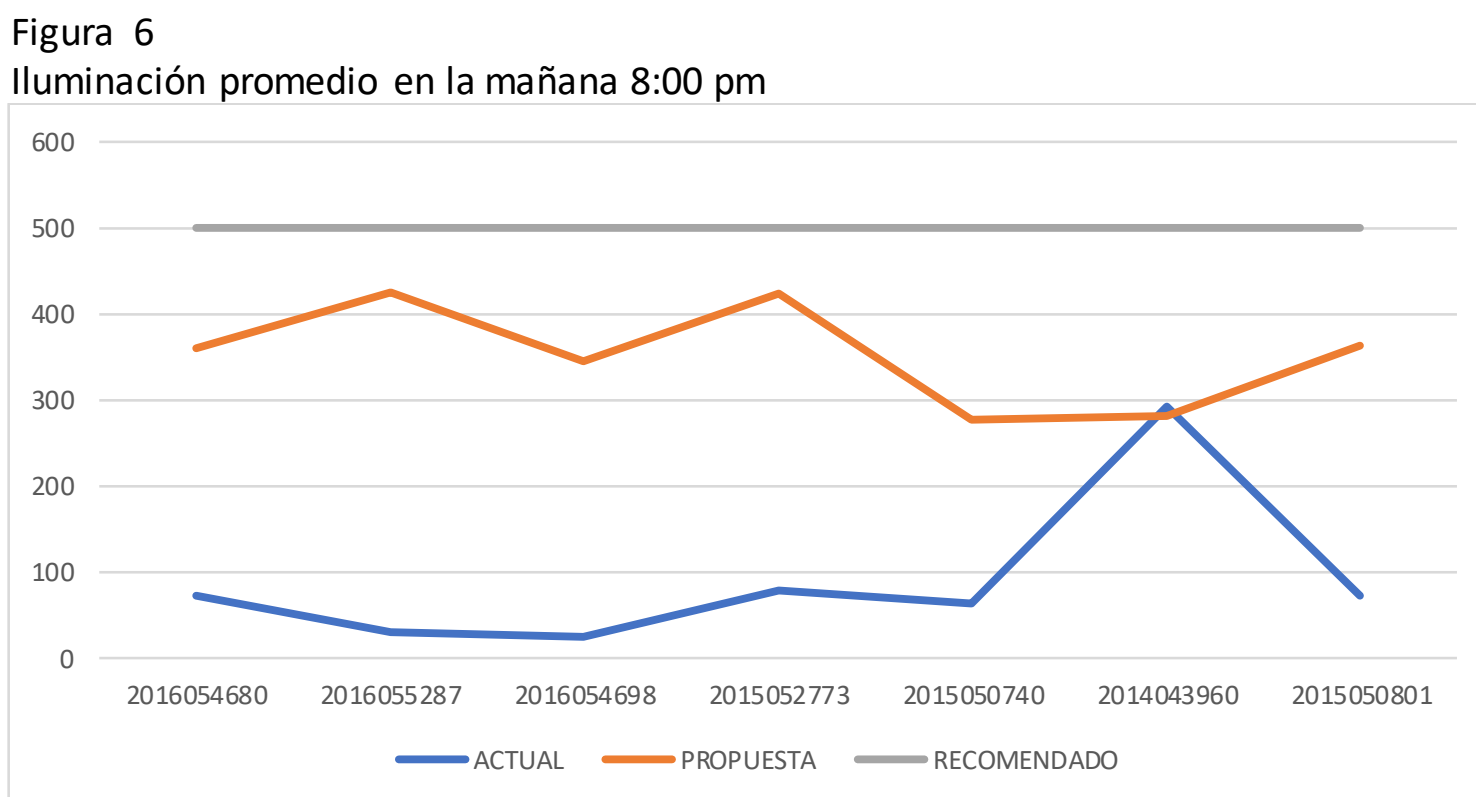

El resultado de los diferentes escenarios simulados demuestra que existiría una mejora de iluminación en el plano de trabajo, aproximándose a los índices recomendados, sin embargo, hace falta un periodo de tiempo más largo para llevar a cabo experimentos de mayor duración y aplicar la propuesta en los ambientes reales para identificar su influencia en las actividades lectivas de los estudiantes universitarios 


\section{DISCUSIÓN:}

Sobre la directriz relacionada a los requerimientos mínimos de iluminación para un área destinada a actividades lectivas, tomando como referencia estándares nacionales e internacionales, según el reglamento nacional de edificaciones y el código técnico de iluminación, el mínimo requerido para áreas destinadas a actividades de aprendizaje es de $5001 x$ y para áreas destinadas a dormitorio de 50lx. Dentro de este panorama encontramos que, las áreas utilizadas para las actividades lectivas en modalidad virtual dentro de las viviendas de los estudiantes, son en la mayoría sus dormitorios, lo cual crea una problemática en el cumplimiento adecuado de iluminación para estos. Sin embargo, elementos como lámparas con iluminación dirigida al plano de trabajo puede garantizar una aproximación a los $5001 x$ en dicho plano y mantener una iluminación adecuada en el dormitorio y de esta manera adaptar los ambientes para albergar las nuevas actividades de ens eñanza virtual. Sin embargo, el estudio demuestra que, de los 7 casos analizados, solo 1 cumple con el uso de este elemento.

En la actualidad existen personas realizando en sus viviendas actividades que hasta antes del periodo de cuarentena desarrollaban en sus lugares de estudio y trabajo. En este contexto se debe considerar medidas de adaptación que puedan garantizar las condiciones mínimas para evitar un efecto negativo en la salud y en el desarrollo de estas actividades. Por lo cual es importante conocer las condiciones de iluminación para el desarrollo de las actividades lectivas en modalidad virtual, así como otros factores sobre temperatura y calidad de aire al interior de las viviendas y así evaluar las condiciones del ambiente al interior de la vivienda. Según un estudio sobre los efectos del ambiente interior de aprendizaje, la iluminación, temperatura, y calidad del aire, son determinantes y pueden influir en el proceso lectivo. (Dangara \& Geraldine, 2019).

\section{CONCLUSIONES}

El estudiante universitario se encuentra en una etapa crítica de aprendizaje, por lo que es necesario brindarle un entorno adecuado que pueda garantizar el correcto desarrollo de sus actividades lectivas. Con el análisis de diferentes estudios, dentro de la revisión bibliográfica, se sustenta la importancia de la iluminación como elemento determinante dentro del ambiente interior de un espacio destinado al aprendizaje. En este contexto, se identificó que la mayoría de los estudiantes encuestados vive en condiciones físicas y espaciales adecuadas para realizar sus estudios en casa, sin embargo, carecen del elemento lumínico suficiente y necesario según las normas técnicas.

Sobre el confort lumínico de los espacios destinados a las actividades lectivas se determinó que la iluminación de estos espacios de estudio está muy por debajo de los requerimientos establecidos en la normativa vigente, con un déficit lumínico del $71.4 \%$. (La norma establece $500 \mathrm{Lx}$ ). Es posible una mejora de condiciones lumínicas a través de la implementación de una luminaria con luz dirigida al plano de trabajo con una potencia $\geq 1800$ lúmenes, 6500K y $18 \mathrm{w}$.

Finalmente, se reconoce que la vivienda, al ser el nuevo escenario de múltiples actividades de trabajo y estudio en modalidad virtual, presenta la necesidad de una correcta adaptación y adecuación de los espacios utilizados para la realización de estas actividades y 
es de suma importancia para un óptimo logro del aprendizaje.

\section{REFERENCIAS BIBLIOGRÁFICAS}

Burruss, W. J. (2001). Adult learning environments: The relationship of light and color in the ambient environment. Journal of Continuing Higher Education, 49(3), 28-33. https://doi.org/10.1080/07377366.2001.10400439

Cachán, C., Carbelo, B., García, M., \& Mateo, P. (2012). Estudio sobre la influencia de la iluminación en el rendimiento escolar. Luces CEI N45, 14-17. http://www.lucescei.com/uploads/tx_ztdownloads/Iluminacion_escolar_Philips_Uni Nebrija.pdf

Dangara, Y., \& Geraldine, C. (2019). Evaluation of the Effect of Learning Environment on Student's Academic Performance in Nigeria.

EM-010, N. T. (2019). Norma técnica em.010 instalaciones eléctricas interiores del reglamento nacional de edificaciones.

Hartstein, L. E., LeBourgeois, M. K., \& Berthier, N. E. (2018). Light correlated color temperature and task switching performance. Preliminary insights. PLOS ONE, 13(8), 1-14. https://doi.org/10.1371/journal.pone.0202973

Lee, M. C., Mui, K. W., Wong, L. T., Chan, W. Y., Lee, E. W. M., \& Cheung, C. T. (2012). Student learning performance and indoor environmental quality (IEQ) in air-conditioned university teaching rooms. Building and Environment, 49(1), 238-244. https://doi.org/10.1016/j.buildenv.2011.10.001

Marchand, G. C., Nardi, N. M., Reynolds, D., \& Pamoukov, S. (2014). The impact of the classroom built environment on student perceptions and learning. Journal of Environmental Psychology, 40, 187-197. https://doi.org/10.1016/j.jenvp.2014.06.009

Niño Rojas, V. M. (2011). Metodologia de la investigacion diseño y ejecucion (1a. Ed.). Bogota: Ediciones de la $u$.

Obeidat, A., \& Al-Share, R. (2012). Quality Learning Environments: Design-Studio Classroom The inscriptions of King Abdullah I Mosque and their relationship with the place View project Quality Learning Environments: Design-Studio Classroom. Asian Culture and History, 4(2). https://doi.org/10.5539/ach.v4n2p165

Samani, S., Samani, S. A., \& Samani, S. A. (2012). The Impact of Indoor Lighting on Students' Learning Performance in Learning Environments: A knowledge internalization perspective. The Influence of Light on Student's Learning Performance. International Journal of Business and Social Science (Vol. 3, Issue 24). www.ijbssnet.com 